\title{
Division of Labour in Self-organised Groups
}

\author{
Roderich Groß ${ }^{1}$, Shervin Nouyan ${ }^{2}$, Michael Bonani ${ }^{1}$, \\ Francesco Mondada ${ }^{1}$, and Marco Dorigo ${ }^{2}$ \\ ${ }^{1}$ LSRO, Ecole Polytechnique Fédérale de Lausanne, Lausanne, Switzerland \\ roderich.gross@ieee.org, \{michael . bonani, francesco.mondada $\}$ @epfl.ch \\ ${ }^{2}$ IRIDIA, CoDE, Université Libre de Bruxelles, Brussels, Belgium \\ \{snouyan, mdorigo\}@ulb.ac.be
}

\begin{abstract}
In social insect colonies, many tasks are performed by higherorder entities, such as groups and teams whose task solving capacities transcend those of the individual participants. In this paper, we investigate the emergence of such higher-order entities using a colony of up to 12 physical robots. We report on an experimental study in which the robots engage in a range of different activities, including exploration, path formation, recruitment, self-assembly and group transport. Once the robots start interacting with each other and with their environment, they selforganise into teams in which distinct roles are performed concurrently. The system displays a dynamical hierarchy of teamwork, the cooperating elements of which comprise higher-order entities. The study shows that teamwork requires neither individual recognition nor inter-individual differences, and as such might contribute to the ongoing debate on the role of such characteristics for the division of labour in social insects.
\end{abstract}

Keywords: cooperation, division of labour, foraging, group transport, hierarchy, path formation, self-organisation, swarm robotics, teamwork.

\section{Introduction}

The field of swarm intelligence draws inspiration from decentralised and selforganising biological systems in general and from the collective behaviour of social insects in particular 112. At present, little is known about the mechanisms that regulate such biological systems, and in particular, about how such mechanisms could enhance the design of swarm intelligence systems. Thus, it is not surprising that the complexity exhibited in current implementations of swarm intelligence systems does neither come close to the complexity of biological systems, nor does it come close to the complexity of systems men built following the more traditional "top-down" approach.

In this paper, we investigate the conditions under which complexity can "emerge" in swarm intelligence systems. One way of measuring complexity is to look at the structural organisation of individuals when performing a task. In an insect colony, various organisational levels can be observed. Both behaviours at the individual level as well as at the colony level have been extensively studied 3. "However, between these two extremes, numerous functional adaptive 
units, or "parts' exist" [4, page 291]. These intermediate-level parts comprise groups and teams.

Teamwork is widely observed in vertebrates. Here, individual recognition is believed to be an important factor 5. Fewer examples of teamwork are known in invertebrates. Oster and Wilson (6]; reviewed in [7]) argue that members of social insect colonies can not form teams as a consequence of their low grade of discrimination: social insects can discriminate "nest mates from aliens, [and] members of one caste as opposed to another" [6, page 151], however, "there is very little evidence that social insects can recognise each other as individuals (but see Tibbetts [8])" 7, page 6]. In contrast, in the recent literature 379, biologists suggest that teams are indeed formed in social insects, and do not require individual recognition. Another aspect that is subject of the ongoing debate is whether inter-individual differences (e.g., members of different castes) are fundamentally required in teamwork [31011].

In the following, we investigate whether tasks that require a complex division of labour fundamentally require individual recognition or inter-individual differences. We illustrate the methods and results of a series of experimental works in which a set of "identical" robots is required to perform a complex, cooperative task. At the beginning of a trial, the robots are randomly scattered in a bounded arena that contains two objects - the prey and the (static) nest. The task is to retrieve the prey to the nest. The following constraints are given:

- the prey requires concurrent, physical handling by multiple robots to be moved,

- each robot's perceptual range is small when compared to the distance between the nest and the prey; moreover, perception is unreliable,

- no robot has any (explicit) knowledge about the environment beyond its perceptual range,

- communication among robots is unreliable and limited to a small set of simple signals that are locally broadcast.

In the following we use the terms groups and teams as defined by Anderson and Franks 11. In particular, a group is a set of individuals that tackle a group task; a team is a set of individuals that tackle a team task. A group task is a task that "requires multiple individuals to perform the same activity concurrently"; a team task is a task that "requires different subtasks to be performed concurrently" (page 535). Furthermore, a partitioned task is "a task that is split into two or more subtasks that are organised sequentially (Jeanne [12] reviewed in Ratnieks and Anderson [13]; Anderson and Ratnieks [14])" 7, page 4]. Anderson and Franks 711, and Anderson and McMillan 15 found that the definition of teamwork, developed primarily from studies of social insects, also applies more generally to societies of other animals, including humans, and robots.

Fig. 1 (left) summarises the division of labour present in our robotic colony. Overall, the robots accomplish a partitioned task comprising three subtasks that are organised sequentially: (i) path formation requires robots to explore the environment and form a path in between the nest and the prey that can be traversed in both directions; (ii) recruitment requires some robots to maintain 

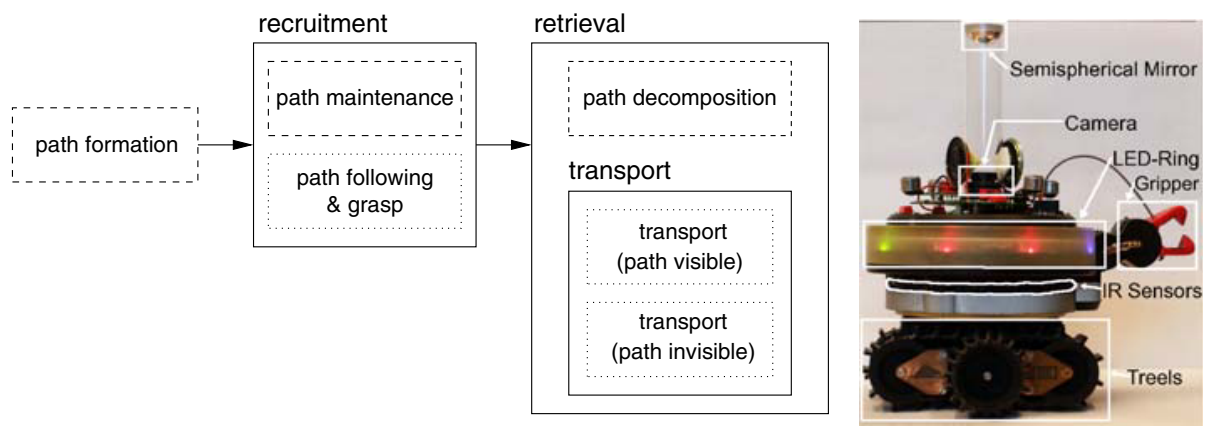

Fig. 1. Left: illustration of the division of labour in our robotic colony that accomplishes a group foraging task. The task is a partitioned task (see arcs). Individual tasks, group tasks and team tasks are framed respectively by dotted, dashed and solid lines. Right: side view of a colony member, the s-bot robot.

the path, while other robots follow the path from the nest to the prey and then grasp either the prey directly or other robots already gripped onto it; (iii) retrieval requires some robots to decompose the path, while other robots transport the prey along the path to the nest (until the prey, or a robot transporting it, is in physical contact with the nest). Path formation itself is a group task, because only a group of robots can establish a path. Similarly, path maintenance and path decomposition are group tasks. Recruitment is a team task, because it requires two different sub-tasks to be performed concurrently-path maintenance and path following $\&$ grasp, where the latter is an individual task. Retrieval is a team task as some robots have to engage in transport, while others, at the same time, have to reside in the path to guide the transport robots towards the nest. Transport can be considered a (nested) team task, as (i) multiple robots are required to transport the prey, and as (ii) the transporting robots, when unable to perceive the path, need to perform distinct actions to avoid that the group transport is ineffective.

The remainder of this paper is organised as follows. In Section 2, we detail the methods, that is, the robot's hardware, the controller and the experimental setup. In Section 3 we present the results. Finally, in Section 4, we discuss the results and conclude the paper.

\section{Methods}

\subsection{Hardware}

We use a robotic system called swarm-bot lying at the intersection between collective and reconfigurable robotics [16]. The system is composed of basic robotic units, called $s$-bots, which are fully autonomous and mobile, and capable of connecting to each other. Fig. 1 (right) shows the physical implementation of the $s$-bot. The robot has a total height of $19 \mathrm{~cm}$ and weighs approximately $700 \mathrm{~g}$. An 


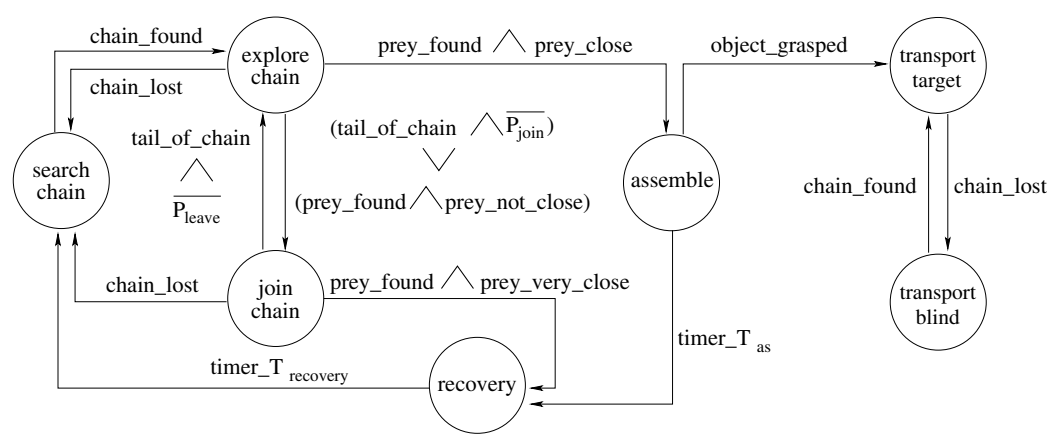

Fig. 2. State diagram of the finite state machine that controls each $s$-bot. Circles represent states (i.e., behaviours). Edge labels specify conditions that trigger transitions between the corresponding states. The initial state is search chain. Variable $\overline{P_{\text {join }}}$ (and $\overline{P_{\text {leave }}}$ ) is True with probability $P_{\text {join }}\left(P_{\text {leave }}\right)$, and False otherwise.

$s$-bot can connect with another by grasping the connection ring with its gripper, and it can receive connections on more than two thirds of its perimeter. The chassis can be rotated in any horizontal direction. This allows $s$-bots, once assembled into a physical entity, to move in a common direction. A 2-D traction sensor, mounted between the $s$-bot's turret and the chassis, measures the mismatch between the direction in which the chassis is trying to move and the direction in which the connected group is trying to move. For the purpose of communication, the s-bot is equipped with an omni-directional camera, four microphones, eight RGB LEDs, and two loudspeakers. For a comprehensive description of the $s$-bot's hardware, see [16].

\subsection{Controller}

The controller consists of a collection of basic behaviours that are implemented using either the motor schema paradigm, neural networks, or simple hand written commands. A comprehensive description of the individual behaviours is available in [17]18/19]20. Following the behaviour-based approach [21] we could comfortably merge all basic behaviours into a common framework, which is illustrated by a state diagram in Fig. 2 ,

Fig. 3 shows a sequence of images taken from the experiment. The $s$-bots are initially located at random positions. If an $s$-bot does neither perceive a (visually connected) chain of $s$-bots nor the nest, it performs a random walk (state search chain). An $s$-bot that finds a chain or the nest follows the perimeter of the encountered structure (state explore chain). The nest can be considered as the root of all chains. When the $s$-bot reaches the tail of a chain, it will join the chain with probability $P_{\text {join }}$ per time step (state join chain). $S$-bots that are part of a chain do not leave it unless they are situated at the chain's tail, in which case they leave it with probability $P_{\text {leave }}$ per time step. The process of 

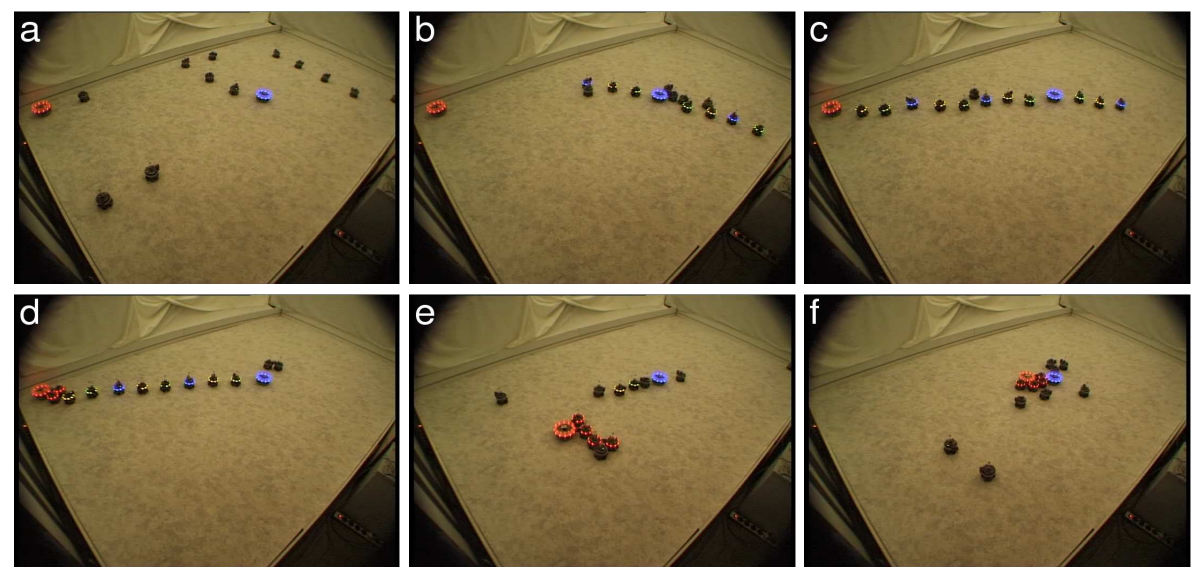

Fig. 3. Sequence of images taken for the trial with group size $N=12 \mathrm{~s}$-bots and distance $D=240 \mathrm{~cm}$ between the nest (blue cylindrical object) and the prey (red cylindrical object). This trial last 15 minutes. For a detailed description, see Section 3

probabilistically joining/leaving a chain is at the basis of the exploration of the environment as it allows the formation of new chains in unexplored areas.

If a chain member perceives the prey it does not leave the chain; thereby the chain becomes stable. If the prey is still far, other $s$-bots can still join to extend the chain in the direction of the prey; otherwise a path connecting the nest to the prey has been formed that can be traversed in both directions. Once a path is formed, it is maintained and in this way automatically recruits other $s$-bots to assemble to the prey (state assemble). S-bots that do not succeed selfassembling within a hard-coded time period, move back to the nest to rest for a while (state recovery). Once a sufficient number of $s$-bots has assembled to the prey, the transport effectively starts; the $s$-bots pull the prey towards the tail of the chain (state transport target). In the event that some $s$-bots can not perceive the path, they use their force sensors to estimate the direction of transport (state transport blind). When the prey reaches the tail of the chain, the corresponding $s$-bot leaves the chain and moves back to the nest to rest for a while (state recovery). In this way the transporting $s$-bots are guided from node to node of the dissolving chain to eventually reach the nest. An $s$-bot leaving the chain to rest at the nest emits a sound signal for a period of $30 \mathrm{~s}$. Transporting $s$-bots respond to this signal by temporarily suspending the transport. This gives the chain $s$-bot sufficient time to move away.

\subsection{Experimental Setup}

The experiments take place in a bounded arena of size $500 \mathrm{~cm} \times 300 \mathrm{~cm}$. The nest is positioned in the centre of the arena. The prey is put at distance $D$ away from the nest towards one of the four corners. $N$-bots are positioned on a grid 
Table 1. Left: number of s-bots required to accomplish sub-tasks path formation $\left(N_{p}\right)$, recruitment $\left(N_{r}\right)$ and retrieval $\left(N_{t}\right)$ for different initial distances ( $D$ in $\mathrm{cm}$ ) between the nest and the prey. Right: overall level of success achieved for setups $(N, D)$ : no success (0), sub-task path formation accomplished (1), sub-task recruitment accomplished (2), and sub-task retrieval accomplished (3). Entries in parentheses denote setups that were not tested as the number of s-bots $N$ is clearly not sufficient to solve the task. Grey levels of cells represent the best achievable level of success: white denotes no success, light grey denotes success level 1, and dark grey denotes success level 3.

\begin{tabular}{lrrrrrrr}
\hline$D$ & 60 & 90 & 120 & 150 & 180 & 210 & 240 \\
\hline$N_{p}$ & 1 & 2 & 3 & 4 & 6 & 7 & 8 \\
$N_{r}$ & 3 & 4 & 5 & 6 & 8 & 9 & 10 \\
$N_{t}$ & 3 & 4 & 5 & 6 & 8 & 9 & 10 \\
\hline
\end{tabular}

\begin{tabular}{ccccccccccc}
\hline$D / N$ & 1 & 2 & 3 & 4 & 5 & 6 & 7 & 8 & 10 & 12 \\
\hline 60 & 1 & 1 & 3 & 3 & 3 & 3 & 3 & 3 & 3 & 3 \\
90 & 0 & 1 & 1 & 3 & 3 & 3 & 3 & 3 & 3 & 3 \\
120 & 0 & 0 & 1 & 1 & 3 & 3 & 2 & 3 & 3 & 2 \\
150 & 0 & 0 & 0 & 1 & 1 & 1 & 3 & 2 & 3 & 3 \\
180 & $(0)$ & $(0)$ & 0 & 0 & 1 & 1 & 1 & 3 & 3 & 3 \\
210 & $(0)$ & $(0)$ & $(0)$ & 0 & 0 & 1 & 1 & 0 & 2 & 2 \\
240 & $(0)$ & $(0)$ & $(0)$ & $(0)$ & $(0)$ & 0 & 0 & 0 & 3 & 3 \\
\hline
\end{tabular}

composed of 60 points uniformly distributed in the arena. The initial position of each $s$-bot is assigned randomly by uniformly sampling without replacement. An $s$-bot's initial orientation is chosen randomly from a set of 12 possible directions.

We study a wide range of experimental setups, with group sizes $N=1,2,3$, 4, 5, 6, 7, 8, 10 and 12, and distances (in cm) $D=60,90,120,150,180,210$ and 240. For each of these 70 setups we conduct a single trial.

The number of $s$-bots required to form a path connecting the prey with the nest depends on the initial distance between the two objects. To calculate lower bounds for the number of $s$-bots, we assume the $s$-bots to be organised in a single chain that is perfectly linear and directed towards the prey. Then, the lower bound values are computed based on the programmed (and measured) distances between adjacent $s$-bots $(27 \mathrm{~cm})$ and between the first chain member and the nest $(30.5 \mathrm{~cm})$, as well as the programmed (and measured) maximum distance of the last chain member from the prey $(38.5 \mathrm{~cm})$. For the accomplishment of the overall task, two additional s-bots are required (at the same time) to engage in transport. The lower bound values so computed are shown in Table 1 (left).

\section{Results}

Table 1 (right) gives an overview of the results. In 46 out of the 70 setups subtask path formation can in principle be accomplished (see light grey cells). In 44 out of the corresponding 46 trials the s-bots succeeded in forming a path. For setups $(N, D)=(5,180)$ and $(6,210)$ a path was formed even though the number of $s$-bots was thought to be insufficient. A path of five (six) s-bots has a maximum predicted length of $177 \mathrm{~cm}(204 \mathrm{~cm})$, which is $3 \mathrm{~cm}(6 \mathrm{~cm})$ less than the distance that needs to be covered, and therefore still within the range of perceptual error of the $s$-bots' cameras. In 33 out of the 46 setups, also sub-tasks recruitment and retrieval can in principle be accomplished (see dark grey cells in Table 1 (right)). In 27 out of these 33 setups, the $s$-bot group was able to do so, thereby the entire task was completed. 

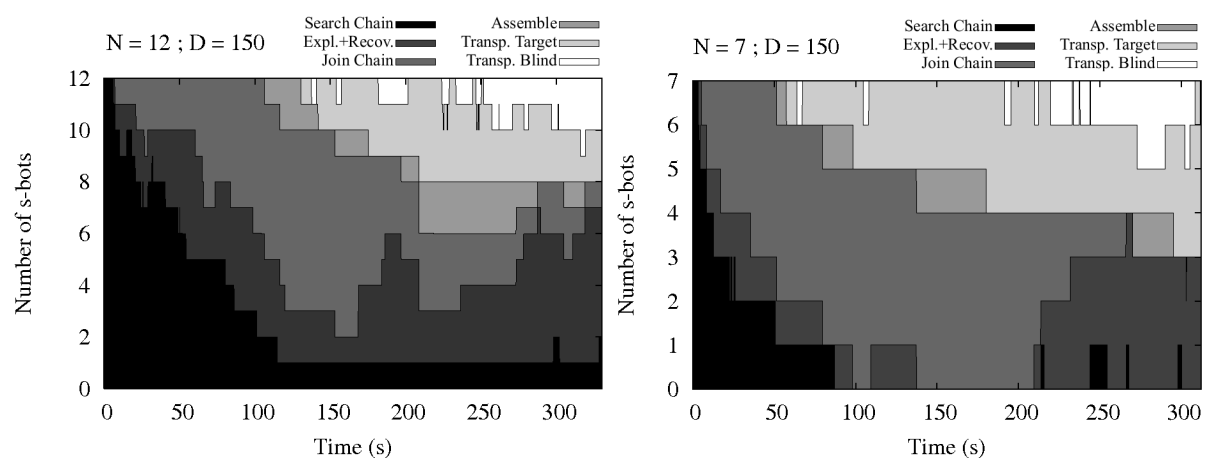

Fig. 4. State dynamics observed in trials for two setups $(N, D)$. The respective grey levels indicate the number of $s$-bots in states search chain, explore chain and recovery, join chain, assemble, transport target and transport blind.

Fig. 4 shows the state dynamics for two trials that are discussed below.

- $(N, D)=(12,150)$ : all $s$-bots start in state search chain. Once the nest has been found, they aggregate into chains. At $t \approx 80 \mathrm{~s}$, a path to the prey consisting of five chain members is established. Even though a path to the prey is formed, other $s$-bots that find the nest self-organise into an additional chain. The formation of the path is not explicitly communicated among the $s$-bots of the group. However, as the $s$-bots in the newly formed chain leave this chain with a constant positive probability, after some time only the chain forming the path remains. At time $t \approx 130 \mathrm{~s}$ a first $s$-bot is recruited and grasps the prey, joined by a second $s$-bot about $15 \mathrm{~s}$ later. While the prey is transported towards the nest, the chain gradually dissolves. During the transport, additional s-bots try to assemble with the pulling structure. Two of them succeed, whereas others fail because the pulling structure is in motion. By looking at the state diagram in Fig. 4 (left), one can see that some of the s-bots engaged in transport are not capable of perceiving the path (see white area). Thus, we have an example where the $s$-bots exhibit a hierarchy of teamwork: the group of $s$-bots that cannot perceive the path needs to interact with the group of $s$-bots that can perceive the path; thereby these groups form a team. This team, which is composed of all transport $s$ bots, can be considered a higher-order entity. It forms part of another team which includes another higher-order entity - the group of s-bots maintaining or decomposing the path. This nested structure is illustrated in Fig. 1] (left).

$-(N, D)=(7,150)$ : at time $t \approx 30 \mathrm{~s}$ a path between nest and prey is already established. At time $t \approx 100 \mathrm{~s}$, two $s$-bots have been recruited and are assembled with the prey. The five remaining $s$-bots are aggregated in the chain forming the path. During the transport, chain members disaggregate once in the immediate vicinity of the prey, and follow the path back 

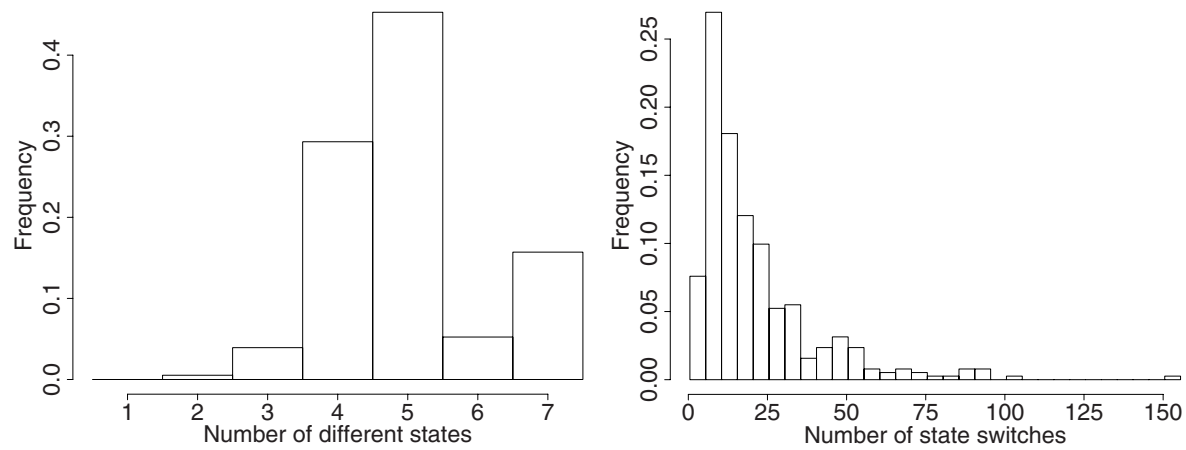

Fig. 5. Left: number of distinct behavioural roles an $s$-bot performed during a trial. Data from all s-bots and all trials. Right: number of times an s-bot changed its behavioural role during a trial. Data from all $s$-bots and all trials.

to the nest to rest. After some time, the very same $s$-bots resume activity, follow the path, and eventually two of them assemble with the pulling structure and participate in transport. This is an example of how the composition of teams can adapt to changes in the workload of the underlying sub-tasks.

Fig. 3 a $-\mathrm{f}$ show a sequence of images taken during the trial with group size $N=12$ and distance $D=240$ (in $\mathrm{cm}$ ). During the path formation phase, two chains are formed concurrently (b), and it takes several rearrangements of the chains until a path is formed. This path consists of a chain of eight $s$-bots (c). Shortly thereafter, two s-bots get recruited and assemble with the prey (d). During retrieval, most of the s-bots of the pulling structure loose sight of the path, which is gradually dissolving, and the prey is moved in the wrong direction (e). However, the path gets re-established by a new s-bot extending the chain in the direction to the prey. As a consequence, the transport resumes and can be completed (f). This is an example of a situation in which teamwork among higher-order entities (such as teams or groups) requires a participating entity to adapt its configuration to unexpected environmental circumstances.

Fig. 5 (left) shows the number of distinct behavioural roles (i.e., states) individual $s$-bots performed during the experiment. In $75 \%$ of the cases, an $s$-bot performed either four or five of the seven roles. This suggests that the $s$-bots are indeed inter-changeable. Only in $4 \%$ of the cases, an $s$-bot performed less than four behaviours during the trial. In $15.7 \%$ of the cases, an $s$-bot performed all seven behaviours.

Fig. 5 (right) shows the number of times an s-bot changed its behavioural role during the trials of our experiments. The most frequently observed number of changes in behaviour belongs to the six to ten changes range. Note, however, that both mean and median number of changes are higher than this range of values (20.9 and 14.5, respectively). 


\section{Discussion}

In this paper, we have presented an experimental study in which a colony of autonomous robots has to solve a complex foraging task. The task requires a range of sub-tasks to be performed including (i) exploration of the environment, (ii) formation of a path between a prey and a nest, (iii) recruitment of nest mates to the prey, (iv) self-assembly into pulling structures, and (v) group transport of the prey back to the nest. Due to the limited abilities of the robots, the accomplishment of the task requires the concurrent activity of at least $i$ robots $(i \in\{3,4,5,6,8,9,10\})$, where $i$ depends on the experimental setting. Moreover, the accomplishment of the task requires division of labour, in other words, the robots need to perform different sub-tasks concurrently. Such constraints are typically not considered in other studies of group foraging systems (e.g., [22 23 24 25 26 20] ), which often make use of some form of global perception or communication, and which often do not require a complex division of labour. In general, we believe that the investigated problem provides a framework that captures the essence of a variety of problems that are addressed at the collective level in social insect colonies.

Inspired by the behaviour of the natural counterparts, we developed a relatively simple, decentralised control algorithm. Although most of our primitive behaviours as well as the overall framework, a finite state machine, were manually designed, we believe that similar types of rules can result from natural or artificial evolutionary processes. In this respect, such systems could be adaptive to changes in the environment.

A series of experimental results from systematic trials with up to twelve physical robots confirm the efficacy of the system. In almost all of the trials where the group size is sufficient to accomplish the overall task, the group succeeded in retrieving the prey to the nest. Video recordings from the experiments are available at http://iridia.ulb.ac.be/supp/IridiaSupp2008-008.

The colony displayed a self-organised and dynamically changing hierarchy of teamwork in which collaboration took also place among high-order entities including groups and teams. The higher-order entities (including the entire system) proved surprisingly robust with respect to the inaccurate and sometimes malfunctioning behaviour of their component modules - parts of a robot such as the tracks, entire robots, and even groups of robots broke down or exhibited unexpected behaviour.

We believe that these experiments are among the most sophisticated examples of self-organisation in robotics to date. The study confirms in a new way that complex forms of division of labour can indeed result from the interactions of individuals that follow relatively simple and local rules. The study also demonstrates that teamwork requires neither individual recognition (the robots we use are inter-changeable) nor inter-individual differences (the robots we use are homogeneous in terms of "morphology" and "brain"), and as such might contribute to the ongoing debate on the role of such characteristics for the division of labour in social insects. 


\section{Acknowledgement}

This work was supported by the Sixth Framework Programme of the European Community in the form of the IST FET project "SWARM-BOTS" (grant no. IST-2000-31010) and of a Marie Curie Intra-European Fellowship (contract no. MEIF-CT-2006-040312), and by the Scientific Research Directorate of the French Community of Belgium in the form of the "ANTS" project, an "Action de Recherche Concertée". It reflects only the authors' views. The European Community is not liable for any use that may be made of the information. Marco Dorigo acknowledges support from the Belgian F.R.S.-FNRS, of which he is a research director. The authors thank Nigel R. Franks for stimulating discussions that helped in the preparation of the manuscript.

\section{References}

1. Bonabeau, E., Dorigo, M., Theraulaz, G.: Swarm Intelligence: From Natural to Artificial Systems. Oxford Univ. Press, New York (1999)

2. Garnier, S., Gautrais, J., Theraulaz, G.: The biological principles of swarm intelligence. Swarm Intelligence 1, 3-31 (2007)

3. Hölldobler, B., Wilson, E.O.: The Ants. Harvard Univ. Press, Cambridge (1990)

4. Anderson, C., McShea, D.W.: Intermediate-level parts in insect societies: Adaptive structures that ants build away from the nest. Insectes Soc. 48, 291-301 (2001)

5. Wilson, E.O.: Sociobiology. Harvard Univ. Press, Cambridge (1975)

6. Oster, G.F., Wilson, E.O.: Caste and ecology in the social insects. Princeton Univ. Press, Princeton (1978)

7. Anderson, C., Franks, N.R.: Teamwork in ants, robots and humans. Adv. Stud. Behav. 33, 1-48 (2004)

8. Tibbetts, E.A.: Visual signals of individual identity in the wasp Polistes fuscatus. Proc. R. Soc. Lond. B 269, 1423-1428 (2002)

9. Franks, N.R.: Teams in social insects: Group retrieval of prey by army ants (Eciton burchelli, Hymenoptera: Formicidae). Behav. Ecol. Sociobiol. 18, 425-429 (1986)

10. Beshers, S.N., Fewell, J.H.: Models of division of labor in social insects. Annu. Rev. Entomol. 46, 413-440 (2001)

11. Anderson, C., Franks, N.R.: Teams in animal societies. Behav. Ecol. 12, 534-540 (2001)

12. Jeanne, R.L.: The evolution of the organization of work in social insects. Monit. Zool. Ital. 20, 119-133 (1986)

13. Ratnieks, F.L.W., Anderson, C.: Task partitioning in insect societies. Insectes Soc. 46, 95-108 (1999)

14. Anderson, C., Ratnieks, F.L.W.: Task partitioning in insect societies: Novel situations. Insectes Soc. 47, 198-199 (2000)

15. Anderson, C., McMillan, E.: Of ants and men: Self-organized teams in human and insect organizations. Emergence 5, 29-41 (2003)

16. Mondada, F., Gambardella, L.M., Floreano, D., Nolfi, S., Deneubourg, J.-L., Dorigo, M.: The cooperation of swarm-bots: Physical interactions in collective robotics. IEEE Robot. Autom. Mag. 12, 21-28 (2005)

17. Groß, R., Bonani, M., Mondada, F., Dorigo, M.: Autonomous self-assembly in swarm-bots. IEEE Trans. Robot. 22, 1115-1130 (2006) 
18. Groß, R., Mondada, F., Dorigo, M.: Transport of an object by six pre-attached robots interacting via physical links. In: Proc. 2006 IEEE Int. Conf. Robot. Autom., pp. 1317-1323. IEEE Comp. Soc. Press, Los Alamitos (2006)

19. Nouyan, S., Campo, A., Dorigo, M.: Path formation in a robot swarm: Selforganized strategies to find your way home. Swarm Intelligence 2 (2008)

20. Tuci, E., Groß, R., Trianni, V., Bonani, M., Mondada, F., Dorigo, M.: Cooperation through self-assembling in multi-robot systems. ACM Trans. on Autonomous and Adaptive Systems 1, 115-150 (2006)

21. Arkin, R.: Behavior-Based Robotics. MIT Press, Cambridge (1998)

22. Kube, C.R., Zhang, H.: Collective robotics: From social insects to robots. Adapt. Behav. 2, 189-218 (1993)

23. Werger, B., Matarić, M.: Robotic food chains: Externalization of state and program for minimal-agent foraging. In: Maes, P., Matarić, M.J., Meyer, J.A., Pollack, J., Wilson, S.W. (eds.) From Animals to Animats 4, Proc. of the 4th Int. Conf. on Simulation of Adaptive Behavior, pp. 625-634. MIT Press, Cambridge (1996)

24. Krieger, M.J.B., Billeter, J.B., Keller, L.: Ant-like task allocation and recruitment in cooperative robots. Nature 406, 992-995 (2000)

25. Kube, C.R., Bonabeau, E.: Cooperative transport by ants and robots. Robot. Auton. Syst. 30, 85-101 (2000)

26. Yamada, S., Saito, J.: Adaptive action selection without explicit communication for multirobot box-pushing. IEEE Trans. Syst., Man, Cybern. C 31, 398-404 (2001) 